\title{
Surface free energy of a hard-sphere fluid at curved walls: Deviations from morphometric thermodynamics ${ }^{+}$
}

Cite as: J. Chem. Phys. 149, 174706 (2018); https://doi.org/10.1063/1.5053929

Submitted: 27 August 2018 . Accepted: 12 October 2018 . Published Online: 05 November 2018

Ruslan L. Davidchack (D), and Brian B. Laird (D)

COLLECTIONS

EP This paper was selected as an Editor's Pick
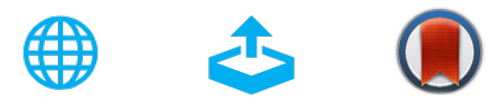

\section{ARTICLES YOU MAY BE INTERESTED IN}

Perspective: Dynamics of confined liquids

The Journal of Chemical Physics 149, 170901 (2018); https://doi.org/10.1063/1.5057759

The quantum mechanics-based polarizable force field for water simulations

The Journal of Chemical Physics 149, 174502 (2018); https://doi.org/10.1063/1.5042658

Nematic order in solutions of semiflexible polymers: Hairpins, elastic constants, and the nematic-smectic transition

The Journal of Chemical Physics 149, 174909 (2018); https://doi.org/10.1063/1.5049630 


\title{
Surface free energy of a hard-sphere fluid at curved walls: Deviations from morphometric thermodynamics
}

\author{
Ruslan L. Davidchack ${ }^{1}$ and Brian B. Laird ${ }^{2,3, a)}$ \\ ${ }^{1}$ Department of Mathematics, University of Leicester, Leicester LE1 7RH, United Kingdom \\ ${ }^{2}$ Department of Chemistry, University of Kansas, Lawrence, Kansas 66045, USA \\ ${ }^{3}$ Freiburg Institute for Advanced Studies, Albert Ludwigs Universität, Albertstraße 19, 79104 Freiburg, Germany
}

(Received 27 August 2018; accepted 12 October 2018; published online 5 November 2018)

\begin{abstract}
We report molecular-dynamics (MD) simulation results for the surface free energy of a hard-sphere fluid at cylindrical and spherical hard walls of different radii. The precision of the results is much higher than that in our previous study [B. B. Laird et al., Phys. Rev. E 86, 060602 (2012)], allowing us to estimate the size of deviations from the predictions of Morphometric Thermodynamics (MT). We compare our results to the analytical expressions for the surface energy as a function of wall radius $R$ and fluid density derived from the White Bear II variant of the density functional theory, as well as to the leading terms of the virial expansion. For the cylindrical wall, we observe deviations from MT proportional to $R^{-2}$ and $R^{-3}$, which are consistent with the available virial expressions. For the spherical wall, while the precision is not sufficient to detect statistically significant deviations from MT, the MD results indicate the range of densities for which the truncated virial expansions are applicable. Published by AIP Publishing. https://doi.org/10.1063/1.5053929
\end{abstract}

\section{INTRODUCTION}

The curvature dependence of the solid-liquid interfacial free energy is crucial to understanding the thermodynamics of crystal nucleation from the melt or solution, ${ }^{1-4}$ as well as for the solvation thermodynamics of nanoparticles. ${ }^{5-7}$

The solvation free energy of a particle with surface $\mathcal{S}$ immersed in a fluid can be written in terms of bulk and surface contributions

$$
F_{\text {solv }}=-P V_{\mathcal{S}}+\gamma_{\mathcal{S}} A_{\mathcal{S}}
$$

where $P$ is the pressure of the surrounding fluid, $V_{\mathcal{S}}$ and $A_{\mathcal{S}}$ are the volume and surface area corresponding to the particle, respectively, and $\gamma_{\mathcal{S}}$ is the surface free energy. Often $\gamma$ is approximated by its value for a planar surface; however, for nanometer scale particles, the contribution of surface curvature to $\gamma$ can be significant. In 3-dimensions, the curvature of the surface can be characterized by specifying the averaged mean and Gaussian curvatures, $\bar{H}$ and $\bar{K}$, respectively,

$$
\begin{gathered}
\bar{H}=\frac{1}{2 A_{\mathcal{S}}} \int_{\mathcal{S}}\left[\frac{1}{R_{1}(\vec{q})}+\frac{1}{R_{2}(\vec{q})}\right] d \mathcal{S}, \\
\bar{K}=\frac{1}{A_{\mathcal{S}}} \int_{\mathcal{S}}\left[\frac{1}{R_{1}(\vec{q}) \cdot R_{2}(\vec{q})}\right] d \mathcal{S},
\end{gathered}
$$

where $R_{1}(\vec{q})$ and $R_{2}(\vec{q})$ are the principal radii at each point $\vec{q}$ on the surface $\mathcal{S}$. For example, for a sphere of radius $R$, the averaged mean and Gaussian curvatures are $1 / R$ and $1 / R^{2}$, respectively, while those for an infinite cylinder of the same radius are $1 / 2 R$ and 0 , respectively.

To describe fluctuating membranes, Helfrich ${ }^{8}$ introduced the so-called Helfrich Hamiltonian in which the free

a)Author to whom correspondence should be addressed: blaird@ku.edu energy of the surface is expanded to quadratic order in $\bar{H}$ and to linear order in $\bar{K}$. This expression can be generalized, expressing the curvature dependence of the surface free energy as a general Taylor series expansion in $\bar{H}$ and $\bar{K}$,

$$
\gamma_{\mathcal{S}}=\sum_{i=0}^{\infty} \sum_{j=0}^{\infty} a_{i j} \bar{H}^{i} \bar{K}^{j} .
$$

In this work, we will be considering the low-order terms of this expansion

$$
\gamma_{\mathcal{S}}=\gamma_{0}+h \bar{H}+\kappa \bar{K}+k \bar{H}^{2}+a_{11} \bar{H} \bar{K}+a_{30} \bar{H}^{3}+\cdots,
$$

with coefficients defined to match the commonly used notations: $\gamma_{0}=a_{00}$ is the surface free energy at a planar wall, $h=a_{10}$ is related to the usual Tolman coefficient $\delta(h=-\delta / 2)$, and $k=a_{20}$ and $\kappa=a_{01}$ are the so-called bending rigidity and Gaussian curvature rigidity, respectively.

For cylindrical and spherical walls, we can also expand $\gamma$ in powers of $1 / R$ (instead of the curvature),

$$
\gamma_{\mathcal{S}}(R)=\sum_{i=0}^{\infty} \frac{\gamma_{i}^{\mathcal{S}}}{R^{i}},
$$

where $\mathcal{S}=\{$ cyl, $\mathrm{sph}\}$ denotes the cylindrical or spherical wall. For a cylindrical surface of radius $R(\bar{H}=1 / 2 R$, $\bar{K}=0$ ), this expansion is related to the Helfrich expansion as follows:

$$
\begin{aligned}
\gamma_{\text {cyl }}(R) & =\gamma_{0}^{\mathrm{cyl}}+\frac{\gamma_{1}^{\mathrm{cyl}}}{R}+\frac{\gamma_{2}^{\mathrm{cyl}}}{R^{2}}+\frac{\gamma_{3}^{\mathrm{cyl}}}{R^{3}}+\mathcal{O}\left(R^{-4}\right) \\
& =\gamma_{0}+\frac{h}{2 R}+\frac{k}{4 R^{2}}+\frac{a_{30}}{8 R^{3}}+\mathcal{O}\left(R^{-4}\right)
\end{aligned}
$$


while for a spherical surface of radius $R\left(\bar{H}=1 / R, \bar{K}=1 / R^{2}\right)$, we have

$$
\begin{aligned}
\gamma_{\mathrm{sph}}(R) & =\gamma_{0}^{\mathrm{sph}}+\frac{\gamma_{1}^{\mathrm{sph}}}{R}+\frac{\gamma_{2}^{\mathrm{sph}}}{R^{2}}+\frac{\gamma_{3}^{\mathrm{sph}}}{R^{3}}+\mathcal{O}\left(R^{-4}\right) \\
& =\gamma_{0}+\frac{h}{R}+\frac{k+\kappa}{R^{2}}+\frac{a_{11}+a_{30}}{R^{3}}+\mathcal{O}\left(R^{-4}\right)
\end{aligned}
$$

Note that, for the spherical wall, expansion of $\gamma$ in powers of $1 / R$ cannot distinguish $k$ and $\kappa$ independently because it includes only their sum, $\gamma_{2}^{\text {sph }}=k+\kappa$.

A much simpler form ${ }^{9}$ of the surface free energy of a fluid at a curved wall is based on Hadwiger's theorem from integral geometry. ${ }^{10}$ In this form, sometimes referred to as Morphometric Thermodynamics (MT), $\gamma$ only depends linearly on the mean and Gaussian curvature, with no higher-order terms,

$$
\gamma_{\mathrm{MT}}=\gamma_{0}+h \bar{H}+\kappa \bar{K} .
$$

Comparing to the Helfrich expression [Eqs. (4) and (5)], the MT expression assumes that all expansion coefficients other than $a_{00}=\gamma_{0}, a_{10}=h$, and $a_{01}=\kappa$ vanish.

A central question in recent research has been the degree to which MT can accurately describe the thermodynamics of curved interfaces. Much of this work has been focused on the standard reference system of a hard-sphere (HS) fluid at a curved hard wall. Initial theoretical work on this system was based on Scaled Particle Theory (SPT). ${ }^{6,11,12}$ For a hard-sphere fluid at a spherical hard wall, SPT yields analytical expressions for the curvature coefficients of $\gamma_{\mathrm{sph}}$,

$$
\begin{aligned}
\gamma_{0}^{\mathrm{SPT}}(\eta) & =\frac{3 \eta(2+\eta)}{2 \pi(1-\eta)^{2}}, \\
h^{\mathrm{SPT}}(\eta) & =\frac{3 \eta}{2 \pi(1-\eta)}, \\
\gamma_{2}^{\mathrm{SPT}}(\eta) & =-\frac{\ln (1-\eta)}{4 \pi},
\end{aligned}
$$

where $\eta$ is the packing fraction, which is proportional to the number density, $\rho, \eta=\pi \rho \sigma^{3} / 6$, where $\sigma$ is the fluid sphere diameter. The SPT expression for the sphere is consistent with MT in that all terms beyond $1 / R^{2}$ for the spherical wall are identically zero. In these and all expressions that follow, we use reduced units in which distances are measured in $\sigma$ and energies in units of $k_{B} T$. Also note that, in defining $\gamma$ for this system, we are using a convention in which the dividing surface is at the point of contact between the wall and the hard-sphere particles, as opposed to another common definition in which the dividing surface corresponds to the centers of the fluid particles at contact. Transformations from one reference to another are straightforward. ${ }^{13,14}$

Bryk et al. ${ }^{15}$ used classical Density Functional Theory (cDFT), specifically Rosenfeld's Fundamental Measure Theory (RFMT), ${ }^{16}$ to examine the curvature dependence of $\gamma$ for a hard-sphere fluid at spherical and cylindrical walls of varying radii. Their results indicate that, within the approximations of RFMT, the coefficient of the linear term in the Taylor series expansion of $\gamma$ in powers of $1 / R$ for the spherical wall is exactly twice (within the error bars) that for the cylindrical wall for packing fractions $\eta$ up to 0.42 . This implies that $h$ is the same for both geometries, consistent with the assumptions of both the Helfrich and MT expansions. In that work, systems with packing fractions greater than 0.42 were not reported as no solutions of the RFMT equations could be found above this

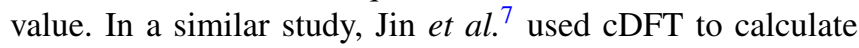
the solvation free energy of nanoscale hard objects of various shapes (cube, cone, etc.) in a hard-sphere fluid. These DFT results were shown to be consistent with MT to high accuracy over the range of hard-sphere packing fractions studied. Reference 7 also compared their results with analytic expressions for the spherical wall generated using the White Bear II (WBII) modification of RFMT, ${ }^{17}$ which, using the current wall convention, can be written in its bulk theory form ${ }^{17}$ as

$$
\begin{aligned}
& \gamma_{0}^{\mathrm{WBII}}=\frac{\eta\left(2+3 \eta-2 \eta^{2}\right)}{\pi(1-\eta)^{2}}-\frac{\ln (1-\eta)}{\pi}, \\
& h^{\mathrm{WBII}}=\frac{\eta(5-\eta)}{2 \pi(1-\eta)}+\frac{\ln (1-\eta)}{\pi}, \\
& \gamma_{2}^{\mathrm{WBII}}=-\frac{\ln (1-\eta)}{4 \pi} .
\end{aligned}
$$

Note that the WBII bulk theory expression is, by construction, like the SPT expression, consistent with MT. Including curvature terms beyond those included in the MT expression requires the consideration of additional scaled variables beyond those included in RFMT and WBII. ${ }^{17}$

Laird et al. ${ }^{18}$ used molecular-dynamics (MD) simulation coupled with Gibbs-Cahn integration ${ }^{19-22}$ to directly evaluate the surface free energy of a hard-sphere fluid at cylindrical and spherical hard walls-the same systems studied by Bryk et al. with cDFT. ${ }^{15}$ The value of the excess volume and the free energy were calculated for wall radii ranging from $\infty$ (planar wall) to $0.5 \sigma$ (identical to the size of the fluid particles). The data for $\gamma$ were fit to polynomial expansions in powers of $1 / R$ to one order greater than the MT predictionup to cubic terms for the spherical wall and quadratic terms for the cylindrical wall. The results show that MT describes the curvature dependence of the systems well up to a packing fraction of about 0.42 ; that is, the value of $h$ for the spherical and cylindrical walls are identical within the statistical error and the first non-MT terms (quadratic and cubic for the cylinder and sphere, respectively) are zero within the error. For $\eta>0.42$, however, deviations from MT are seen to grow rapidly as the fluid approaches the freezing transition.

Using cDFT or virial expansions, several groups have recently reported theoretical results that predict deviations from MT in hard-sphere fluid/curved wall systems even at relatively low packing fractions. Blokhuis, ${ }^{23}$ using the RFMT version of cDFT, indicated small deviations even for small packing fractions $(\eta<0.1)$; however, the deviations seen were on the order of the statistical error in the simulations, so no conclusions as to the accuracy of the cDFT calculations relative to simulation could be reached. Similar results were obtained by Reindl et al. ${ }^{14}$ using a cDFT based on a second-virial expansion. By calculating virial coefficients for the hard-sphere fluid at spherical and cylindrical walls, Urrutia $^{13}$ derived low-order expansions of $h, \kappa$, and the bending rigidity $k$ in powers of the packing fraction $\eta$. Most notable is the conclusion that the bending rigidity is small, 
but non-zero, even at low $\eta$-in direct contradiction of the assumptions of MT. A similar conclusion was reached by Hansen-Goos ${ }^{24}$ who also suggested possible modifications to RFMT cDFT to include non-MT corrections. All of these recent theoretical studies cannot be verified using the previously published simulation data ${ }^{18}$ because the predicted deviations are on the order of the statistical error of the simulations.

In this work, we perform a series of very high resolution MD simulations on a hard-sphere fluid at spherical and cylindrical hard walls, which, coupled with Gibbs-Cahn integration, give values of the surface free energy $\gamma$ with statistical errors that are small enough to properly test the theoretical predictions. We concentrate here on lower densities $(\eta \lesssim 0.3$ ) because the deviations at higher packing fractions were well resolved in our previous work ${ }^{18}$ and higher resolution data would not change the conclusions. In Sec. II, we discuss the current status of virial expansions for coefficients in Eqs. (7) and (8). Section III presents the details of the MD simulation and analysis methods followed by a discussion of the major results of the study in Sec. IV. In Sec. V, we summarize and conclude.

\section{VIRIAL COEFFICIENTS FOR HARD-SPHERE FLUIDS AT CURVED HARD WALLS}

The coefficients in Eqs. (7) and (8) are all functions of the fluid packing fraction $\eta$ and can be expanded in a virial series in powers of $\eta$ with the generic form

$$
\alpha(\eta)=\sum_{j=0}^{\infty} \alpha_{j} \eta^{j},
$$

where $\alpha_{j}$ are the virial coefficients for quantity $\alpha(\eta)$.

For the hard-sphere fluid at a curved wall, the first few virial coefficients have been calculated independently by Urrutia $^{13}$ and Hansen-Goos, ${ }^{24}$

$$
\begin{aligned}
& h=\frac{3}{2 \pi}\left[\eta+\eta^{2}+\left(\frac{8}{35}+\frac{27 \sqrt{3}}{16 \pi}\right) \eta^{3}\right]+\mathcal{O}\left(\eta^{4}\right), \\
& \kappa=\frac{1}{4 \pi}\left[\eta-\eta^{2}\right]+\mathcal{O}\left(\eta^{3}\right), \\
& k=\frac{3}{8 \pi} \eta^{2}+\mathcal{O}\left(\eta^{3}\right) .
\end{aligned}
$$

The non-zero value of $k$ in these expressions indicates that there should be measurable deviations from MT even at low density.

For completeness, the analytically known virial terms for the planar wall are

$$
\gamma_{0}=\frac{3}{\pi} \eta+\frac{15}{2 \pi} \eta^{2}+\frac{759}{70 \pi} \eta^{3}+\mathcal{O}\left(\eta^{4}\right)
$$

with the higher-order terms known numerically from Monte Carlo evaluation of cluster integrals. ${ }^{25,28}$ Recalling Eqs. (7) and (8) for the cylindrical and spherical geometries, $\gamma_{0}^{\text {cyl }}=\gamma_{0}^{\text {sph }}$, first order terms $\gamma_{1}^{\text {cyl }}$ and $\gamma_{1}^{\text {sph }}$ are equal to $h / 2$ and $h$, respectively, and the second order term for the cylindrical wall $\gamma_{2}^{\text {cyl }}=k / 4$. For other higher-order terms, Urrutia ${ }^{13}$ gives

$$
\begin{aligned}
\gamma_{2}^{\mathrm{sph}} & =k+\kappa=\frac{1}{4 \pi}\left[\eta+\frac{1}{2} \eta^{2}+\left(\frac{81 \sqrt{3}}{16 \pi}-\frac{289}{105}\right) \eta^{3}\right]+\mathcal{O}\left(\eta^{4}\right) \\
\gamma_{3}^{\mathrm{cyl}} & =\frac{1}{8} a_{30}=-\frac{3}{64 \pi} \eta^{2}+\mathcal{O}\left(\eta^{3}\right) \\
\gamma_{3}^{\mathrm{sph}} & =a_{30}+a_{11}=\frac{9 \sqrt{3}}{160 \pi^{2}} \eta^{3}+\mathcal{O}\left(\eta^{4}\right)
\end{aligned}
$$

\section{SIMULATION AND ANALYSIS METHODS}

As in our previous work, ${ }^{18}$ we calculate the surface free energy of a hard-sphere fluid at cylindrical and spherical walls using the Gibbs-Cahn adsorption equation adapted for fluids interacting with static walls, ${ }^{22}$

$$
\left(\frac{\partial \gamma}{\partial P}\right)_{T}=v_{\mathrm{ex}}
$$

where $v_{\text {ex }}$ is the excess surface volume defined as

$$
v_{\mathrm{ex}}=\frac{1}{A N_{f}}\left|\begin{array}{cc}
V & N \\
V_{f} & N_{f}
\end{array}\right|=\frac{1}{A}\left(V-V_{f} \frac{N}{N_{f}}\right) .
$$

In Eq. (25), $A$ is the surface area of the cylinder or sphere, $V$ and $N$ are the volume and number of particles, respectively, of a region containing the interface, and $V_{f}$ and $N_{f}$ are the corresponding quantities for a region entirely within the bulk fluid. ${ }^{22}$ The excess interfacial volume for cylindrical and spherical walls of radius $R$ can be related to the number density profile $\rho(r)$ by

$$
v_{\mathrm{ex}}=\frac{1}{R^{i}} \int_{R}^{\infty}\left[1-\frac{\rho(r)}{\rho_{f}}\right] r^{i} d r,
$$

where $i=1$ and 2 for the cylindrical and spherical geometries, respectively, and $\rho_{f}=N_{f} / V_{f}$ is the bulk fluid density.

Because the surface free energy vanishes in the limit of zero pressure, Eq. (24) can be integrated to give

$$
\gamma(P)=\int_{0}^{P} v_{\mathrm{ex}}\left(P^{\prime}\right) d P^{\prime} .
$$

Because of the difficulty in performing hard-sphere MD simulations efficiently at constant pressure, $v_{\mathrm{ex}}$ is calculated in our simulations as a function of the packing fraction $\eta=\pi \rho_{f} / 6$ so that

$$
\gamma(\eta)=\int_{0}^{\eta} v_{\mathrm{ex}}\left(\eta^{\prime}\right)\left(\frac{\partial P}{\partial \eta^{\prime}}\right)_{T} d \eta^{\prime} .
$$

The derivative in the integrand of Eq. (28) is calculated using the Kolafa-Labík-Malejevskí (KLM)-low Equation of State (EOS). ${ }^{27}$ This EOS reproduces simulation pressures to very high precision, even at high pressures near the freezing density, and so its use introduces errors smaller than the statistical errors of the present calculations.

The hard-sphere (HS) systems at different densities interacting with a flat wall, as well as with cylindrical and spherical walls of different radii, were simulated using the moleculardynamics algorithm of Rapaport, ${ }^{26}$ with suitable modifications to include interactions with the curved walls. Density profiles as functions of the distance from the wall were measured and the excess volume, $v_{\mathrm{ex}}$, was calculated from the density profiles as described in the supplementary material of Ref. 18. For 
the flat wall systems, the simulation setup and the results have recently been published elsewhere. ${ }^{28}$

For the cylindrical wall system, we calculated results for the wall radii $R=0.4,0.5,0.6,0.7,1.0,1.5,2.0,5.0$, and 10.0 in reduced units as defined earlier. The simulation box had dimensions $L_{x}, L_{y}$, and $L_{z}$ in the three Cartesian directions and the cylindrical wall axis was set parallel to the $x$ axis. Systems with $L_{x} \approx 10,20$, and 40 were simulated. We also used different system sizes in the $y$ and $z$ directions: $L_{y}-2 R=L_{z}$ $-2 R \approx 18$ and 26 for $\eta<0.06$ and 26 and 38 for $\eta>0.06$. Periodic boundary conditions were applied in all directions. We did not observe statistically significant dependence of the results on $L_{y}$ and $L_{z}$, so the reported results for $v_{\mathrm{ex}}$ are the averages over these simulations. The reason for this is that we used relatively large values of $L_{y}=L_{z}$ in order to have a large region of fluid away from the wall, where the density profile oscillations due to the presence of the wall are insignificant compared to the statistical error (which is at a distance of about 7.5 from the wall for the highest packing fraction $\eta=0.32$ studied in this work). At the same time, we observed a statistically significant dependence on $L_{x}$ at low densities, which decreased with increasing $\eta$ and became statistically insignificant for $\eta>0.06$. To account for the observed size dependence at $\eta<0.06$, we calculated the weighted leastsquares best fit to a straight line as a function of $L_{x}^{-1}$ of the results at $L_{x}=10,20$, and 40 and used the straight line intercept with $L_{x}^{-1}=0$ for the reported results, together with the corresponding estimated $95 \%$ confidence interval in the position of the intercept. For $\eta>0.06$, the reported results are averages of the simulation results with $L_{x}=20$ and 40 (we did not extend the simulations of the system with $L_{x}=10$ to larger $\eta$ ).

For the spherical wall system, we calculated results for the wall radii $R=1.0,1.5,2.0,5.0$, and 10 . We used a cubic simulation box with sizes $L_{x}-2 R=L_{y}-2 R=L_{z}-2 R \approx 18$ and 26 for $\eta<0.1$ and 26 and 38 for $\eta>0.1$. We did not observe a statistically significant size dependence of the results, so the reported results are averages of the results for systems with different system sizes. As in our previous work, ${ }^{18}$ the excess volume for the system with a spherical wall with $R=0.5$ is calculated by recognizing that the "wall particle" is the same size as the fluid particles, so $\rho(r)$ (and thus $v_{\mathrm{ex}}$ ) can be determined from the radial distribution function (RDF) of a simulation of the bulk hard-sphere fluid. We simulated systems with dimensions $L_{x}=L_{y}=L_{z} \approx 25$ and 38 . We did not observe a statistically significant size dependence of the results, so the reported results are averages of the results for systems with the two system sizes.

Once $v_{\mathrm{ex}}$ for the cylindrical and spherical walls were obtained as discussed above, $\gamma$ was calculated by numerical integration using Eq. (28). Because the data are unevenly spaced as a function of $\eta$, we use the trapezoidal rule. In Ref. 18, we reduced the numerical error in the integration by first subtracting from the integrand the SPT expression. The exact SPT expression for $\gamma$ was then added back in after the numerical integration. This reduced the curvature of the integrand so that the numerical integration error was smaller than the estimated statistical error in $\gamma$. For the present calculations, with a substantially smaller statistical error, this is not sufficient. Instead, we subtract off a more accurate analytical expression-namely, that derived from the WBII bulk theory [Eqs. (13)-(15)]. Subtracting the expression for $\partial \gamma / \partial \eta$ generated from the WBII equations from the integrand for both the spherical and cylindrical cases before integration lowers the magnitude and curvature of the integrand so that an upper bound of the numerical integration error is lower than the estimated statistical error. The value of $\gamma$ is then determined by adding back in the WBII expression for $\gamma(R, \eta)$.

\section{RESULTS AND DISCUSSION}

The high-resolution simulation results for the excess volume for the hard-sphere fluid at both spherical and cylindrical walls, plotted as a function of the packing fraction $\eta$ for all radii studied, are summarized in Fig. 1 . The value at $\eta=0$ was obtained using the exact low density limit of $\rho(R)$ resulting in

$$
v_{\mathrm{ex}}(R, \eta=0)= \begin{cases}\frac{1}{2}+\frac{1}{4 R}+\frac{1}{24 R^{2}} & (\text { spherical wall) } \\ \frac{1}{2}+\frac{1}{8 R} & \text { (cylindrical wall). }\end{cases}
$$

The error estimates for the data in Fig. 1 are significantly smaller than in Ref. 18 and cannot be resolved visually in the figure.

Using the data shown in Fig. 1 and Eq. (28), we calculate $\gamma(R, \eta)$ using the methods described in Sec. IIII. The results are summarized in Fig. 2 as functions of wall radius $R$ and packing fraction $\eta$ for both the spherical and cylindrical walls. From these data, we see a strong curvature dependence of $\gamma$, which increases with increasing curvature as measured by the inverse radius $1 / R$. The numerical values of $v_{\mathrm{ex}}(R, \eta)$ and $\gamma(R, \eta)$ with error bars are tabulated in the supplementary material.

To assess the validity of the MT expression [Eq. (9)], which states that $\gamma$ for the cylinder and the sphere should be strictly linear and quadratic functions, respectively, of $1 / R$, the data for $\gamma(R, \eta)$ for both wall types were fit to a cubic polynomial in $1 / R$ using weighted least-squares linear regression (also sometimes referred to as cubic regression). ${ }^{29}$ In Fig. 3, the linear coefficient, $h$, as defined in Eq. (5), is plotted for both the cylindrical and spherical walls. Consistent with Helfrich and MT expansions, the values of $h$ for these two wall

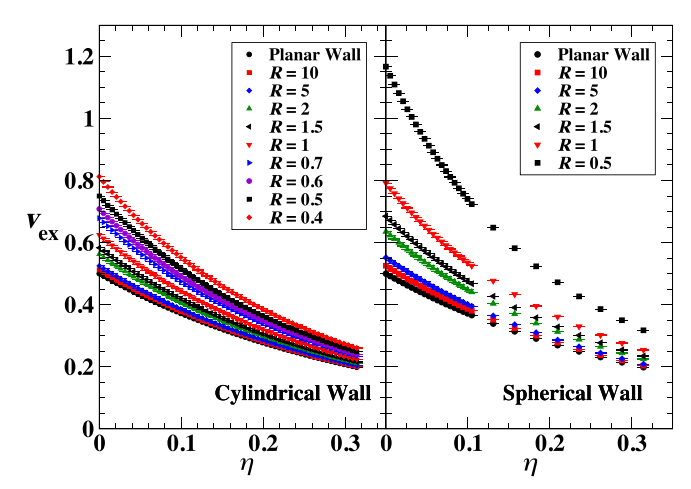

FIG. 1. Excess volume, $v_{\mathrm{ex}}(R, \eta)$, for a hard-sphere fluid at cylindrical (left) and spherical (right) hard walls as functions of the cylinder (sphere) radius, $R$, and the packing fraction, $\eta$. 


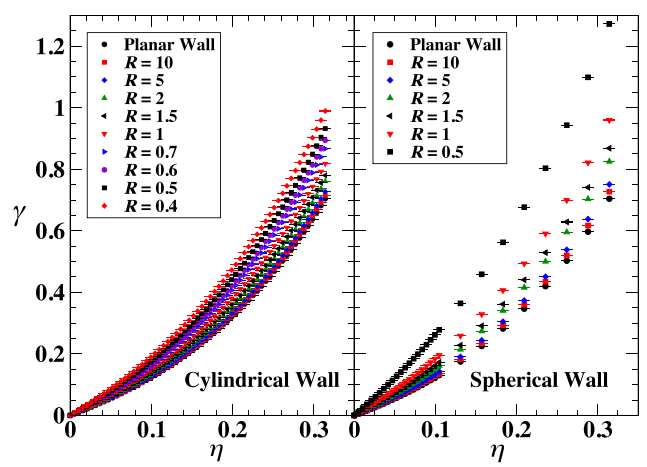

FIG. 2. The surface free energy $\gamma(R, \eta)$ of a hard-sphere fluid at cylindrical (left) and spherical (right) hard walls as a function of the wall radius $R$ and of packing fraction $\eta$.

types are equal within the error estimates for all $\eta$ studied in this work. To see this more clearly, the difference between the two $h$ values, $h_{\text {sph }}-h_{\text {cyl }}$, is plotted in the inset of Fig. 3 . Also plotted in Fig. 3 are the truncated virial expansion for $h$ from Eq. (17) and the expression in Eq. (14) from the WBII bulk theory. The virial expression agrees with the simulation well, up to about $\eta \approx 0.2$, but even at $\eta=0.3$, the deviations are only a few percent. The WBII expression is even closer.

The simulation results for the full set of MT coefficients for the cylinder $\left(h_{\text {cyl }}\right)$ and the sphere $\left(h_{\text {sph }}\right.$ and $\left.\gamma_{2}^{\text {sph }}=k+\kappa\right)$ are shown in Fig. 4. For the spherical wall, the value of $\gamma_{2}^{\text {sph }}$ is considerably smaller than the linear term $h$. Also, the virial expression for $\gamma_{2}^{\text {sph }}$ [Eq. (21)] is in excellent agreement with the simulation data over the range of packing fractions studied. Figure 4 also shows the virial expansion result for $\kappa$ alone [Eq. (18)—obtained by subtracting the virial expansion for $k$ determined from the cylindrical geometry from that for $\gamma_{2}^{\text {sph }}$ ]. At low packing fractions $(\eta<0.1)$, the contributions of $k$ to $\gamma_{2}^{\text {sph }}$ are negligible on a relative scale (consistent with MT); however, at the higher packing fractions, the contribution of

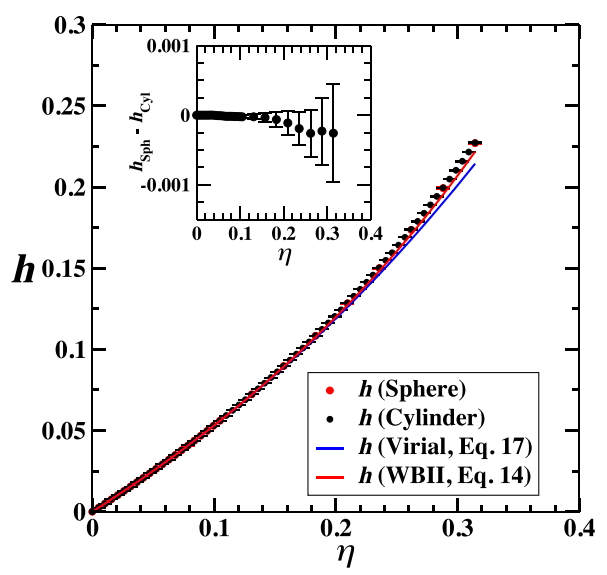

FIG. 3. The coefficient $h$ for the hard-sphere fluid at spherical and cylindrical walls as functions of packing fraction $\eta$ determined from the simulation data using least-squares cubic regression in $1 / R$. For comparison, also shown are the truncated virial expansion expression for $h$ from Eq. (17) and the WBII expression from Eq. (14). Because the values for the spherical and cylindrical walls appear the same at the resolution of this plot, the difference between them is plotted in the inset.

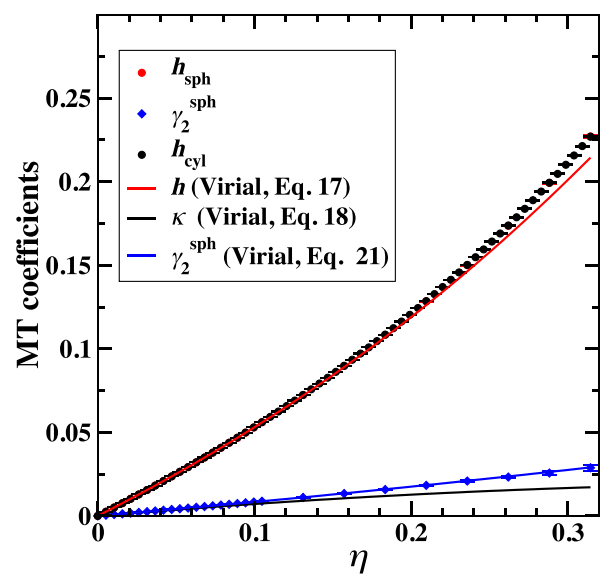

FIG. 4. The MT coefficients for the hard-sphere fluid at spherical ( $h_{\mathrm{sph}}$ and $\left.\gamma_{2}^{\text {sph }}=k+\kappa\right)$ and cylindrical $\left(h_{\text {cyl }}\right)$ walls as functions of packing fraction $\eta$ determined from the simulation data using least-squares cubic regression in $1 / R$. For comparison, also shown are the corresponding truncated virial expansion expressions, including that for $\kappa$.

$k$ to $\gamma_{2}^{\text {sph }}$ is much larger (up to $32 \%$ at the highest packing fractions studied), indicating potentially significant deviations from MT predictions when non-spherical geometries (such as ellipsoids) are considered.

We now turn specifically to higher-order non-MT terms for the cylindrical and spherical geometries. For the hardsphere fluid at a cylindrical wall, the first such term is the quadratic coefficient $\gamma_{2}^{\text {cyl }}$ in Eq. (7), related to the bending rigidity $k$ in Helfrich's expansion, Eq. (5), $\gamma_{2}^{\text {cyl }}=k / 4$. We denote this bending rigidity $k_{\mathrm{cyl}}$, as this quantity is obtained from the cylindrical wall data using weighted least-squares linear regression in powers of $1 / R$ at each value of $\eta$. The results are shown in Fig. 5, where we plot the results obtained both with the linear regression up to $1 / R^{3}$ (weighted cubic fit) and up to $1 / R^{4}$ (weighted quartic fit). As we can see, the two results differ somewhat, with the statistically significant difference (which we associate with the non-overlapping error

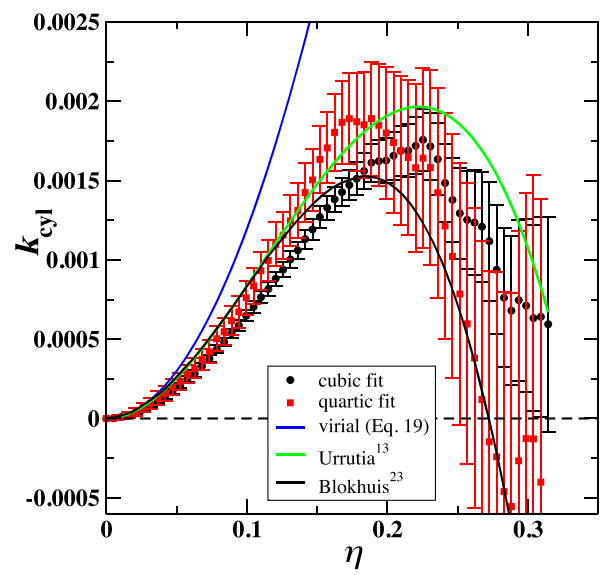

FIG. 5. The bending rigidity coefficient, $k_{\text {cyl }}$, for the hard-sphere fluid at a cylindrical wall as a function of packing fraction $\eta$. The solid black circles and red squares are the results obtained from the cubic and quartic weighted regression fit in powers of $1 / R$. The blue line is the truncated virial expression in Eq. (19), the green line is the expression proposed by Urrutia, ${ }^{13}$ and the black line is the RFMT cDFT result of Blokhuis. ${ }^{23}$ 
bars) in the range $0.1<\eta<0.2$. This indicates that within the precision of our MD computations, we detect a relatively small, but statistically significant, contribution from the $1 / R^{4}$ term in the expansion in Eq. (7). For comparison, we show the leading term of the virial expansion for the bending rigidity in Eq. (19), which agrees with the simulation results only for $\eta<0.02$. At the same time, the empirical expression proposed by Urrutia, ${ }^{13} k(\eta)=3 \eta^{2}(1-3 \eta) /(8 \pi)$ and the RFMT cDFT result of Blokhuis ${ }^{23}$ show very good agreement with the simulation results.

The results for the cubic curvature coefficient for the cylindrical wall, $\gamma_{3}^{\text {cyl }}$, are shown in Fig. 6. Again, we see somewhat different results for the cubic and quartic weighted regression fits, but both indicate that $\gamma_{3}^{\text {cyl }}$ is negative for most of the range of $\eta$ values studied in this work. This is consistent with the first term of the virial expression, Eq. (22), which is also negative. (Note that Ref. 13 contains a typo in which the coefficient $\gamma_{3}^{\text {cyl }}$ is reported as positive when it actually has the opposite sign..$^{30}$ )

For the spherical wall, the first non-MT term is $\gamma_{3}^{\text {sph }}$ in the expansion of $\gamma$ in powers of $1 / R$, Eq. (8). The virial expression for this term was given earlier in Eq. (23). The simulation data for $\gamma_{3}^{\text {sph }}$, obtained using weighted cubic regression in powers of $1 / R$, are plotted in Fig. 7. Even with the high precision of the current simulation results, $\gamma_{3}^{\text {sph }}$ cannot be distinguished from zero within the statistical error. This is also verified by the observation that the quadratic regression in powers of $1 / R$ fits the spherical wall simulation results for all $\eta$ studied. As can be seen in Fig. 7, the simulation data are consistent with the virial expansion results up to about $\eta=0.05$, with small deviations in the region between packing fractions of 0.05 and 0.1 . One cannot rule out the possibility, though, that these deviations might disappear when higher-order virial expansion coefficients are considered.

In analogy to Eq. (5) for $\gamma$, we can also expand the excess volume in a Helfrich expansion, or, similar to Eqs. (7) and (8), in the series in powers of $1 / R$ for the cylindrical and spherical walls, respectively,

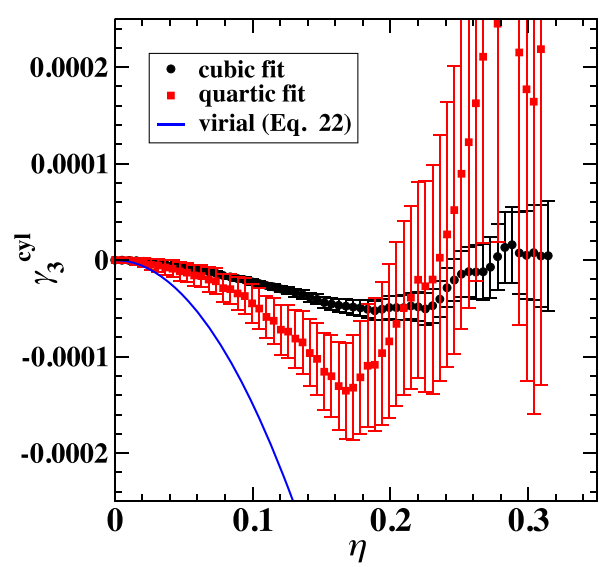

FIG. 6. The cubic curvature coefficient, $\gamma_{3}^{\text {cyl }}$, for the hard-sphere fluid at a cylindrical wall as a function of packing fraction $\eta$. The black circles and red squares are the results obtained from the cubic and quartic weighted regression fit in powers of $1 / R$. The blue solid line shows the truncated virial expansion in Eq. (22).

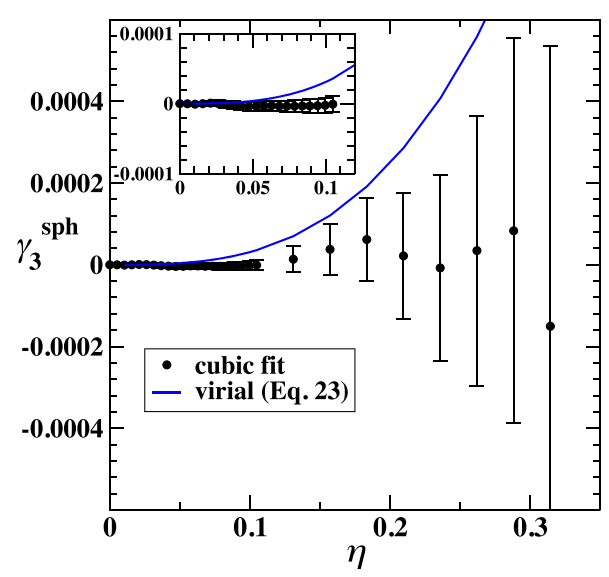

FIG. 7. The cubic curvature coefficient, $\gamma_{3}^{\text {sph }}$, for the hard-sphere fluid at a spherical wall as a function of packing fraction $\eta$. The solid circles represent the results for the current simulations. The blue line shows the virial expansion up to third order in $\eta$. The inset shows the same data at low packing fractions.

$$
\begin{aligned}
v_{\mathrm{ex}} & =v_{0}+h_{v} \bar{H}+\kappa_{v} \bar{K}+k_{v} \bar{H}^{2}+\cdots \\
& =v_{0}+\frac{v_{1}}{R}+\frac{v_{2}}{R^{2}}+\frac{v_{3}}{R^{3}}+\cdots \\
& =v_{0}^{\mathrm{cyl}}+\frac{h_{v}^{\mathrm{cyl}}}{2 R}+\frac{k_{v}^{\mathrm{cyl}}}{4 R^{2}}+\frac{v_{3}^{\mathrm{cyl}}}{R^{3}}+\cdots(\text { cyl }) \\
& =v_{0}^{\mathrm{sph}}+\frac{h_{v}^{\mathrm{sph}}}{R}+\frac{\kappa_{v}+k_{v}^{\mathrm{sph}}}{R^{2}}+\frac{v_{3}^{\mathrm{sph}}}{R^{3}}+\cdots(\mathrm{sph}) .
\end{aligned}
$$

If MT holds for the excess volume, then we would have $h_{v}^{\text {cyl }}=h_{v}^{\text {sph }}, k_{v}^{\text {cyl }}=k_{v}^{\text {sph }}=0$, and zero for all coefficients of order $1 / R^{3}$ and higher. To assess this, we fit the simulation results for $v_{\text {ex }}$ shown in Fig. 1 for the cylindrical and spherical walls to cubic and quartic polynomials in $1 / R$ using weighted least-squares linear regression. The linear term corresponding to $h_{v}$ is plotted in Fig. 8 for both the spherical and the cylindrical walls. As shown in the inset of Fig. 8, the values of $h_{v}$ for

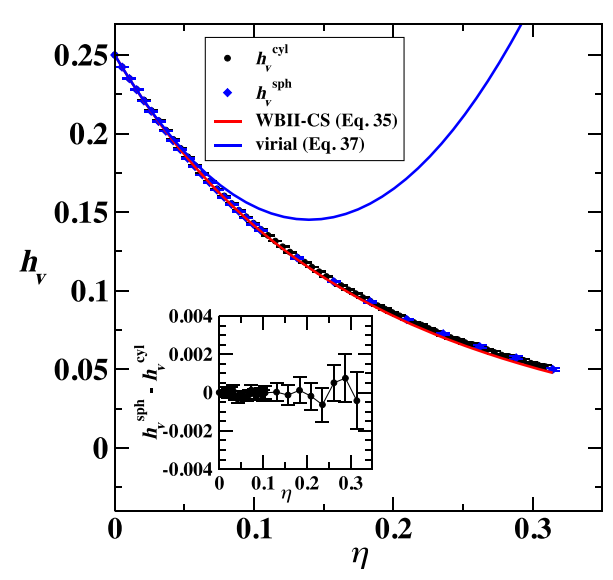

FIG. 8. The coefficients $h_{v}$ for the hard-sphere fluid at spherical $\left(h_{v}^{\mathrm{sph}}\right)$ and cylindrical $\left(h_{v}^{\text {cyl }}\right)$ walls as functions of packing fraction $\eta$ obtained via weighted cubic regression in powers of $1 / R$ of the excess volume simulation results for the spherical and cylindrical walls, respectively. For comparison, also shown is the WBII expression for $h_{v}$ from Eq. (35) and the virial expansion [Eq. (37)]. Because the values for the spherical and cylindrical walls appear the same at the resolution of this plot, the difference between them is plotted in the inset. 
both wall types are identical within the error bars, consistent with the predictions of MT. Also shown in Fig. 8 is the expression for $h_{v}$ derived from the WBII expression for $h$ [Eq. (14)] and using the fact that

$$
v_{\mathrm{ex}}(\eta)=\left(\frac{\partial \gamma}{\partial \eta}\right) /\left(\frac{\partial P}{\partial \eta}\right),
$$

where for $P(\eta)$ we use the Carnahan-Starling (CS) equation of state from which the WBII expression is derived

$$
P_{\mathrm{CS}}=\frac{6 \eta\left(1+\eta+\eta^{2}-\eta^{3}\right)}{\pi(1-\eta)^{3}} .
$$

The WBII-CS expression for $h_{v}$ is

$$
h_{v}^{\mathrm{WBII-CS}}=\frac{(1-\eta)^{2}\left(3+\eta^{2}\right)}{12\left(1+4 \eta+4 \eta^{2}-4 \eta^{3}+\eta^{4}\right)},
$$

which is seen to be in excellent agreement with the simulation results.

The virial expression for $h_{v}$ can be determined from the virial expansion for $h$, combined with Eq. (33) and the usual virial expansion for the hard-sphere pressure,

$$
P=\frac{6}{\pi} \eta+\frac{24}{\pi} \eta^{2}+\frac{60}{\pi} \eta^{3}+\cdots,
$$

yielding

$$
h_{v}=\frac{1}{4}-\frac{3}{2} \eta+\left(\frac{327}{70}+\frac{81 \sqrt{3}}{64 \pi}\right) \eta^{2}+\cdots .
$$

This virial expansion is also plotted in Fig. 8 and we note that the range of $\eta$ values for which the virial series for $h_{v}$ agrees with the simulation results is significantly smaller than that for $h$.

In Fig. 9, we plot the simulation data for the excessvolume expansion coefficient $v_{2}^{\mathrm{sph}}=k_{v}^{\mathrm{sph}}+\kappa_{v}$ for the hardsphere fluid at a spherical wall. Also shown are the WBII-CS expression

$$
\left(k_{v}+\kappa_{v}\right)^{\mathrm{WBII}-\mathrm{CS}}=\frac{(1-\eta)^{3}}{24\left(1+4 \eta+4 \eta^{2}-4 \eta^{3}+\eta^{4}\right)},
$$

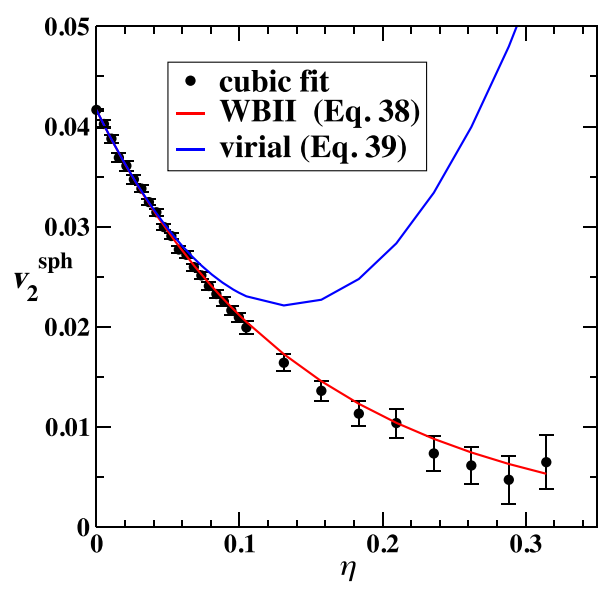

FIG. 9. The curvature coefficient $v_{2}^{\mathrm{sph}}=k_{v}^{\mathrm{sph}}+\kappa_{v}$ for the excess volume as a function of packing fraction $\eta$ from the simulations (black circles). Also shown are lines corresponding to the WBII and virial expansion expressions.

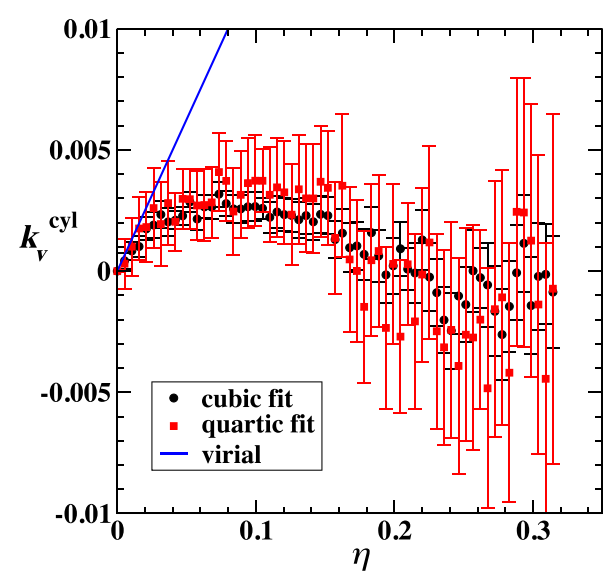

FIG. 10. The first non-MT term $\left(k_{v}^{\mathrm{cyl}}\right)$ for the excess volume of the hardsphere fluid at a cylindrical hard wall as a function of packing fraction $\eta$. The solid (black) circles and (red) squares are the results obtained from the cubic and quartic weighted regression fit in powers of $1 / R$, respectively. Also shown is the first term, $\eta / 8$, of the virial expansion.

derived in the same way as Eq. (35), and the virial expression, given by

$$
k_{v}+\kappa_{v}=\frac{1}{24}-\frac{7}{24} \eta+\left(\frac{207}{280}+\frac{81 \sqrt{3}}{128 \pi}\right) \eta^{2}+\cdots
$$

As with the results for $h_{v}$, the WBII expression is in excellent agreement with the simulation.

The first non-MT term for the excess volume for the cylindrical wall, $k_{v}^{\text {cyl }}$, is shown in Fig. 10. This quantity does exhibit a significant deviation from zero, even at low packing fractions. The results obtained using weighted linear regression in powers of $1 / R$ up to cubic and quartic terms are consistent within the error bars. Also plotted is the first virial expansion term for this quantity, which is $\eta / 8$. Within the error bars, the simulation data in the linear regime at small $\eta$ are consistent with this first virial term. Higher-order virial coefficients would be needed for comparisons with the simulation results at higher packing fractions.

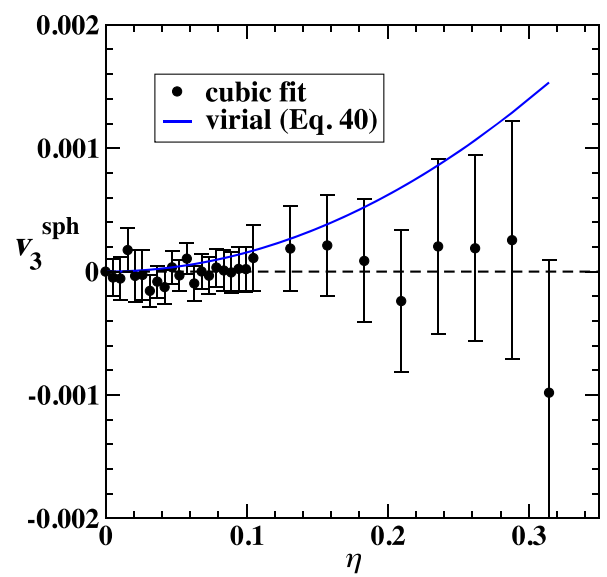

FIG. 11. The first non-MT term $\left(v_{3}^{\mathrm{sph}}\right)$ from the simulations (filled circles) for the excess volume of a hard-sphere fluid at a spherical hard wall as a function of packing fraction $\eta$. Also shown is the leading term of the virial expansion from Eq. (40). 
The first non-MT term from the simulations for the excess volume for the spherical wall, $v_{3}^{\text {sph }}$, is shown in Fig. 11. Within our error estimates, this term is not significantly different from zero, so even at this high resolution simulation results for the spherical wall, it is not possible to verify the existence of deviations from MT. Also plotted is the first virial expansion term for this quantity,

$$
v_{3}^{\mathrm{sph}}=\frac{9 \sqrt{3}}{320 \pi} \eta^{2}+\cdots
$$

At low $\eta$, where this expression should be valid, it is consistent with the simulation results; however, making any further conclusions would require the accurate calculation of higherorder virial coefficients and even higher precision simulation data.

\section{SUMMARY}

We have performed a series of high-resolution moleculardynamics (MD) simulations to determine the excess volume, $v_{\text {ex }}$, and surface free energy, $\gamma$, of a hard-sphere fluid at spherical and cylindrical curved walls of varying radii. The motivation for these high-precision simulations is to attempt to resolve deviations at low-packing from the so-called Morphometric Thermodynamics (MT), which predicts that the surface free energy (and $v_{\text {ex }}$ ) can be written as a linear combination of the average mean and Gaussian wall curvatures. In our previous work, ${ }^{18}$ our simulation identified strong deviations from MT at high packing, but deviations at low packing fraction, predicted by recent theoretical studies using classical Density Functional Theories (cDFT) $)^{14,23}$ and virial expansion techniques, ${ }^{13,24}$ were not resolvable within the statistical error of those simulations. For the spherical (cylindrical) walls studied here, MT predicts that all terms in a Taylor series for $\gamma\left(\right.$ or $v_{\mathrm{ex}}$ ) in powers of $1 / R$ beyond the $1 / R^{2}(1 / R)$ term are identically zero. By calculating $\gamma$ as a function of both the packing fraction $\eta$ and the wall radius $R$ for both spherical and cylindrical walls and fitting the subsequent $\gamma(R, \eta)$ [or $v_{\mathrm{ex}}(R, \eta)$ ] to a cubic or quartic polynomial in $1 / R$ at each fixed $\eta$, we were able to test the assumptions of MT and compare any deviations to the recent theoretic predictions.

Our results show that, even with the higher resolution, the MT coefficient linear in the mean curvature, $h$, for both the spherical and cylindrical walls is identical within the statistical error, consistent with the predictions of MT (and the more general Helfrich expansion). For the cylindrical system, the non-MT coefficients of $1 / R^{2}$ and $1 / R^{3}$ for both $\gamma$ and $v_{\mathrm{ex}}$ are found to deviate from zero with statistical significance, even at low packing fractions, and are consistent with the virial coefficients of Urrutia, ${ }^{13}$ as well as the DFT calculations of Blokhuis. ${ }^{23}$ For the spherical system, however, even these very high precision results are insufficient to resolve deviations from the MT predictions, with the calculated non-MT higher order term (cubic in $1 / R$ ) being zero within the error bars. Given the central role the hard-sphere fluid plays as a reference system for more physically realistic systems, the results obtained here imply that similar deviations from MT are likely to be observed for more complex interactions as well.

\section{SUPPLEMENTARY MATERIAL}

See supplementary material for files containing the numerical data from the simulations for the surface free energy, $\gamma$, and excess volume, $v_{\mathrm{ex}}$, for the hard-sphere fluid at both spherical and cylindrical curved walls.

\section{ACKNOWLEDGMENTS}

B.B.L. acknowledges support from the National Science Foundation (NSF) under Grant No. CHE-1465226. B.B.L. would like to thank the Freiburg Institute of Advanced Studies for hosting him during much of the data analysis and writing that went into the manuscript. R.L.D. did part of the work during a study leave granted by the University of Leicester. This research used the ALICE High Performance Computing Facility at the University of Leicester. We also wish to thank Professor Roland Roth and Dr. Hendrik Hansen-Goos for helpful discussions.

${ }^{1}$ A. Cacciuto, S. Auer, and D. Frenkel, J. Chem. Phys. 119, 7467-7470 (2003).

${ }^{2}$ D. T. Wu, L. Granasy, and F. Spaepen, MRS Bull. 29, 945 (2004).

${ }^{3}$ X.-M. Bai and M. Li, J. Chem. Phys. 124, 124707 (2006).

${ }^{4}$ R. L. Davidchack, R. Handel, J. Anwar, and A. V. Brukhno, J. Chem. Theory Comput. 8(7), 2383-2390 (2012).

${ }^{5}$ K. Lum, D. Chandler, and J. D. Weeks, J. Phys. Chem. 103, 4570 (1999).

${ }^{6}$ J. R. Henderson, J. Chem. Phys. 116, 5039-5045 (2002).

${ }^{7}$ Z. Jin, J. Kim, and J. Wu, Langmuir 28, 6997-7006 (2012).

${ }^{8}$ W. Helfrich, Z. Naturforsch., C 28, 693-703 (1973).

${ }_{9}^{9}$ P.-M. König, R. Roth, and K. R. Mecke, Phys. Rev. Lett. 93, 160601 (2004).

${ }^{10} \mathrm{H}$. Hadwiger, Vorlesungen ueber Inhalt, Oberflaeche und Isoperimetrie (Springer, Berlin, 1957)

${ }^{11}$ H. Reiss, H. L. Frisch, E. Helfand, and J. L. Lebowitz, J. Chem. Phys. 32, 119 (1960).

${ }^{12} \mathrm{H}$. Reiss, "Scaled particle methods in the statistical thermodynamics of fluids," in Advances in Chemical Physics, edited by I. Prigogine (John Wiley \& Sons, Inc., 1965), Vol. 9, pp. 1-84.

${ }^{13}$ I. Urrutia, Phys. Rev. E 89, 032122 (2014).

${ }^{14}$ A. Reindl, M. Bier, and S. Dietrich, Phys. Rev. E 91, 022406 (2015).

${ }^{15}$ P. Bryk, R. Roth, K. R. Mecke, and S. Dietrich, Phys. Rev. E 68, 031602 (2003).

${ }^{16}$ Y. Rosenfeld, Phys. Rev. Lett. 63, 980-983 (1989).

${ }^{17}$ H. Hansen-Goos and R. Roth, J. Phys.: Condens. Matter 18, 8413-8425 (2006).

${ }^{18}$ B. B. Laird, A. Hunter, and R. L. Davidchack, Phys. Rev. E 86, 060602 (2012).

${ }^{19}$ J. W. Cahn, "Thermodynamics of solid and fluid surfaces," in Interfacial Segregation edited by W. C. Johnson and J. M. Blakely (ASM International, International Materials Park, OH, 1979), pp. 3-23.

${ }^{20}$ T. Frolov and Y. Mishin, Phys. Rev. B 79, 045430 (2009).

${ }^{21}$ B. B. Laird, R. L. Davidchack, Y. Yang, and M. Asta, J. Chem. Phys. 131, 114110 (2009).

${ }^{22}$ B. B. Laird and R. L. Davidchack, J. Chem. Phys. 132, 204101 (2010).

${ }^{23}$ E. M. Blokhuis, Phys. Rev. E 87, 022401 (2013).

${ }^{24}$ H. Hansen-Goos, J. Chem. Phys. 141, 171101 (2014).

${ }^{25}$ J. H. Yang, A. J. Schultz, J. R. Errington, and D. A. Kofke, J. Chem. Phys. 138(13), 134706 (2013).

${ }^{26}$ D. C. Rapaport, The Art of Molecular Dynamics Simulation, 2nd ed. (Cambridge University Press, New York, 2004).

${ }^{27}$ J. Kolafa, S. Labík, and A. Malijevský, Phys. Chem. Chem. Phys. 6, 23352340 (2004).

${ }^{28}$ I. E. Paganini, R. L. Davidchack, B. B. Laird, and I. Urrutia, J. Chem. Phys. 149, 014704 (2018).

${ }^{29}$ In cubic weighted linear least squares regression, the "best" fit of a set of $N$ data points $\left(x_{i}, y_{i} ; i=1, \ldots, N\right)$ to a functional form $y(x)=a+b x+c x^{2}+d x^{3}$ is obtained by minimizing the residual function $F(a, b, c, d)=\sum_{i=1}^{N}\left[y_{i}-\right.$ $\left.y\left(x_{i}\right)\right]^{2} / \delta_{i}^{2}$ (where the $\delta_{i}^{2}$ are the $95 \%$ confidence level variances in $y_{i}$ ) with respect to the fitting parameters $a, b, c$, and $d$.

${ }^{30}$ I. Urrutia, private communication (2018). 